Gynäkologe 2018·51:1064

https://doi.org/10.1007/s00129-018-4364-8

Online publiziert: 9. November 2018

(c) Springer Medizin Verlag $\mathrm{GmbH}$, ein Teil von Springer Nature 2018

CrossMark

Jens H. Stupin

Klinik für Gynäkologie, Charité-Universitätsmedizin Berlin, Campus Virchow-Klinikum, Berlin, Deutschland

\title{
Erich Saling - Eine lebende Legende
}

\section{Leserbrief zu}

Vetter K (2018) Erich Saling - Vater der Perinatalmedizin. Gynäkologe 51:734-736. https://doi.org/10.1007/s00129-018-4297-2

Herrn Prof. Klaus Vetter ist für die umfassende und auch sehr persönliche Würdigung der klinisch-wissenschaftlichen Leistungen und der Person des „Vaters der Perinatalen Medizin“ aufrichtig zu danken.

Als junger Kollege, der sich seit langem mit der wissenschaftlichen Biographie Erich Salings beschäftigt und Mitherausgeber des Standardwerks The Beginnings of Perinatal Medicine [1] ist, kann ich die Worte des Verfassers nur unterstreichen.

Wir sollten uns vor Augen halten, dass es in keinem anderen Gebiet der Medizin noch möglich ist, dem Begründer persönlich zu begegnen.

Mit der ersten Fetalblutanalyse, durchgeführt am 21.06.1961 in der Städtischen Frauenklinik Berlin-Neukölln, leitete Saling die „kopernikanische Wende" in der Geburtshilfe ein, durch die das Kind in den Mittelpunkt gestellt wurde. Nach der Publikation des Buches Das Kind im Bereich der Geburtshilfe [2] begann ein bis heute anhaltender Aufschwung dieser Medizin mit vielen Innovationen: von der fetalen Überwachung sub partu, dem pränatalen Ultraschall bis hin zur Fetalchirurgie.

Beim Blick in das Programm des 62. Kongresses der Deutschen Gesellschaft für Gynäkologie und Geburtshilfe (DGGG) vom 31.10.-03.11.2018 fiel auf, dass der international hoch geschätzte „Vater der Perinatalen Medizin“, immerhin Ehrenmitglied und seit 2006 Träger der höchsten Auszeichnung der DGGG, der Carl-Kaufmann-Medail- le, nicht mit einem wissenschaftlichen Beitrag, z.B. zu dem ihn seit mehr als 25 Jahren beschäftigenden brisanten Thema „Frühgeburtenvermeidung“ u.a. durch Selbstvorsorge der Schwangeren, beteiligt war.

Leider eine verpasste Chance, vor allem für viele junge Kolleginnen und Kollegen, eine "lebende Legende“ und ein Vorbild hautnah im wissenschaftlichen Austausch erleben zu können.

\section{Korrespondenzadresse}

\section{Dr. med. Jens H. Stupin}

Klinik für Gynäkologie, Charité-Universitätsmedizin Berlin, Campus VirchowKlinikum

Augustenburger Platz 1, 13353 Berlin, Deutschland

jens.stupin@charite.de

Interessenkonflikt. J.H. Stupin gibt an, dass kein Interessenkonflikt besteht.

\section{Literatur}

1. Saling E, Dräger M, Stupin JH (Hrsg) (2014) The Beginnings of Perinatal Medicine. De Gruyter, Berlin, München, Boston

2. Saling E (1966) Das Kind im Bereich der Geburtshilfe. Thieme, Stuttgart 